\title{
Universiteit
}

Leiden

The Netherlands

\section{Cost-effectiveness of feedback-informed psychological treatment: evidence from the IAPT-FIT trial}

Delgadillo, J.; McMillan, D.; Gilbody, S.; Jong, K. de; Lucock, M.; Lutz, W.; ... ; Ali, S.

\section{Citation}

Delgadillo, J., McMillan, D., Gilbody, S., Jong, K. de, Lucock, M., Lutz, W., ... Ali, S. (2021). Cost-effectiveness of feedback-informed psychological treatment: evidence from the IAPTFIT trial. Behaviour Research And Therapy, 142. doi:10.1016/j.brat.2021.103873

Version: Publisher's Version

License: $\quad$ Licensed under Article 25fa Copyright Act/Law (Amendment Taverne)

Downloaded from: https://hdl.handle.net/1887/3250370

Note: To cite this publication please use the final published version (if applicable). 


\title{
Cost-effectiveness of feedback-informed psychological treatment: Evidence from the IAPT-FIT trial
}

\author{
Jaime Delgadillo $^{\mathrm{a}, *}$, Dean McMillan ${ }^{\mathrm{b}}$, Simon Gilbody ${ }^{\mathrm{b}}$, Kim de Jong ${ }^{\mathrm{c}}$, Mike Lucock ${ }^{\mathrm{d}}$, \\ Wolfgang Lutz ${ }^{\mathrm{e}}$, Julian Rubel ${ }^{\mathrm{f}}$, Elisa Aguirre ${ }^{\mathrm{g}}$, Shehzad Ali ${ }^{\mathrm{b}, \mathrm{h}}$ \\ ${ }^{a}$ Clinical Psychology Unit, Department of Psychology, University of Sheffield, United Kingdom \\ ${ }^{\mathrm{b}}$ Department of Health Sciences and Hull York Medical School, University of York, United Kingdom \\ ${ }^{\mathrm{c}}$ Institute of Psychology, Leiden University, Netherlands \\ ${ }^{\mathrm{d}}$ Centre for Applied Research in Health, University of Huddersfield, UK \\ e Department of Psychology, University of Trier, Germany \\ ${ }^{\mathrm{f}}$ Department of Psychology, Justus-Liebig-University Giessen, Germany \\ ${ }^{g}$ North East London National Health Service (NHS) Foundation Trust, UK \\ ${ }^{\mathrm{h}}$ Department of Epidemiology and Biostatistics, Western University, Canada
}

\section{A R T I C L E I N F O}

\section{Keywords:}

Psychotherapy

Feedback-informed treatment

Cost effectiveness

Economic analysis

\begin{abstract}
A B S T R A C T
Background: Feedback-informed treatment (FIT) involves using computerized routine outcome monitoring technology to alert therapists to cases that are not responding well to psychotherapy, prompting them to identify and resolve obstacles to improvement. In this study, we present the first health economic evaluation of FIT, compared to usual care, to enable decision makers to judge whether this approach represents a good investment for health systems.

Methods: This randomised controlled trial included 2233 patients clustered within 77 therapists who were randomly assigned to a FIT group $(n=1176)$ or a usual care control group $(n=1057)$. Treatment response was monitored using patient-reported depression (PHQ-9) and anxiety (GAD-7) measures. Therapists in the FIT group had access to a computerized algorithm that alerted them to cases that were "not on track", compared to normative clinical data. Health service costs included the cost of training therapists to use FIT and the cost of therapy sessions in each arm. The incremental cost-effectiveness of FIT was assessed relative to usual care, using multilevel modelling.

Results: FIT was associated with an increased probability of reliable symptomatic improvement by 8.09 percentage points (95\% CI: $4.16 \%-12.03 \%$ ) which was statistically significant. The incremental cost of FIT was $£ 15.17$ (95\% CI: $£ 6.95$ to $£ 37.29$ ) per patient and was not statistically significant. The incremental costeffectiveness ratio (ICER) per additional case of reliable improvement was $£ 187.4$ (95\% CI: $£ 126.7$ to $£ 501.5$ ); this confidence interval shows that the relative cost-effectiveness is between FIT being a dominant strategy (i.e. more effective and also cost-saving) to FIT being more effective at a modest incremental cost to the health system.

Conclusions: The FIT strategy increases the probability of reliable improvement in routine clinical practice and may be associated with a small (but uncertain) incremental cost. FIT is likely to be a cost-effective strategy for mental health services.
\end{abstract}

\section{Introduction}

Feedback-informed treatment (FIT) involves alerting therapists to cases that are not responding well to therapy, prompting them to identify and resolve obstacles to improvement in a timely way (Lambert,
Hansen, \& Finch, 2001). This is done by routinely monitoring patients' symptoms or functioning using standardised self-reported measures before each session. These measures are entered into a computer system that compares them to measures observed in other similar cases. Patients who show poor progress compared to similar cases are classed as "not on

\footnotetext{
* Corresponding author. Clinical Psychology Unit, University of Sheffield, Floor F, Cathedral Court, 1 Vicar Lane, Sheffield, S1 2LT, United Kingdom.

E-mail address: jaime.delgadillo@nhs.net (J. Delgadillo).
} 
track" by an automated algorithm, and this information is fed back to the therapist. Therapists are thus prompted to discuss potential difficulties with the patient, and to seek advice from clinical supervisors to identify possible solutions. Meta-analyses of controlled trials of psychotherapy with adult participants conclude that using feedback technology can improve treatment outcomes (e.g., Knaup, Koesters, Schoefer, Becker, \& Puschner, 2009; Lambert, Whipple, \& Kleinstäuber, 2018; Shimokawa, Lambert, \& Smart, 2010) and this effect is more robust in cases classed as "not on track" (Kendrick et al., 2016). Overall, quantitative data from over 50 studies in this area, including controlled trials and quasi-experimental studies, indicates that FIT is associated with improved treatment outcomes and reduced dropout rates, although incremental effects sizes are small relative to usual psychological care (De Jong et al., 2021).

Recent studies in primary care mental health services have indicated that feedback technology also potentially enhances the efficiency of treatment, resulting in similar clinical outcomes but in fewer-thanaverage treatment sessions (Delgadillo et al., 2017; Janse, De Jong, Van Dijk, Hutschemaekers, \& Verbraak, 2017). This evidence, however, comes from quasi-experimental studies without contemporaneous control groups, and it stands in contrast to meta-analyses of controlled trials which did not find significant differences in treatment duration between FIT vs. control conditions (e.g., see Knaup et al., 2009). Nevertheless, even if FIT does not reduce the average duration of treatment, it could still be potentially more cost-effective if it results in improved treatment outcomes. However, no previous studies in this field have conducted health economic analyses to examine the cost-effectiveness of FIT. The present study aimed to fill this gap in the literature by presenting the first health economic evaluation of a FIT treatment system implemented in the Improving Access to Psychological Therapies (IAPT) programme in the United Kingdom.

\section{Methods}

\subsection{Design and setting}

The present paper reports secondary analyses of a clinical trial dataset. The aim of this study was to evaluate the comparative costeffectiveness of FIT versus usual psychological care, using data from the IAPT-FIT Trial. This was a multi-site, pragmatic, cluster randomised controlled trial conducted in England. The trial included 2233 patients treated by 77 psychological therapists across eight healthcare organisations. Participating therapists (cluster-level) were randomly assigned to a FIT group ( $n=1176$ patients within $k=39$ therapists) or a usual care control group $(n=1057 ; \mathrm{k}=38)$. The trial was statistically powered to detect a small effect size difference between groups, using a clustered data structure with patients nested within therapists. Randomisation was carried out by an independent researcher using a computerized random sequence generator, with stratification by service to attain balanced assignment within each trial site. The trial was preregistered in the international register of controlled trials (ISRCTN12459454) and approved by an independent NHS Research Ethics Committee (Ref: 15/LO/2200). Further details about the study design, inclusion and exclusion criteria, consort diagram, sample size calculation and statistical analyses are available elsewhere (Delgadillo et al., 2018).

\subsection{Interventions}

Usual care involved standardised, protocol-driven stepped care interventions recommended by clinical guidelines for common mental disorders (National Institute for Health and Care Excellence, 2011). These included guided self-help, cognitive behavioural therapy, interpersonal psychotherapy, and counselling for depression, which were delivered in accordance with treatment-specific competency standards (e.g., National IAPT Team, 2015; Roth \& Pilling, 2008). These interventions were delivered by qualified psychological practitioners under regular clinical supervision, equivalent to $1 \mathrm{~h}$ of supervision per week of full-time practice. Therapists in the FIT group had access to a computerized algorithm that compared patients' depression and anxiety symptoms to normative clinical data, classifying patients as "on track" or "not on track", and which was represented graphically using expected treatment response curves (Delgadillo et al., 2017). Therapists assigned to the FIT group attended a single 6.5-h training workshop which guided them on how to interpret feedback graphs, how to monitor and discuss this feedback with patients at the start of every therapy session, and they were instructed to prioritise "not on track" cases for discussion with clinical supervisors. All other aspects of treatment (e.g., protocol-driven interventions, frequency of clinical supervision) were standardised across the FIT and the control group.

\subsection{Measures}

Patients routinely completed three validated patient-reported outcome measures using paper-based questionnaires which were reviewed by all therapists (in the FIT and control groups) at the start of each therapy session. The PHQ-9 is a measure of depression symptoms, where each of 9 questions is rated from 0 to 3, yielding an overall severity score between 0 and 27 (Kroenke, Spitzer, \& Williams, 2001). A cut-off of $\geq 10$ has been recommended to screen for clinically significant depression symptoms, with adequate sensitivity (88\%) and specificity (88\%). A difference of $\geq 6$ points between measurements is indicative of statistically reliable change (Richards \& Borglin, 2011). The reliability of the PHQ-9 in the present sample was excellent (Cronbach's alpha = .92). The GAD-7 is a 7-item measure of generalized anxiety disorder; each item is also rated between 0 and 3 , with a total severity score between 0 and 21 (Kroenke, Spitzer, Williams, Monahan, \& Löwe, 2007). A cut-off score $\geq 8$ is recommended to screen for clinically significant anxiety problems including GAD and other anxiety disorders, with adequate sensitivity (77\%) and specificity ( $82 \%$ ). A change of $\geq 5$ points has been recommended to assess reliable change (Richards \& Borglin, 2011). The reliability of the GAD-7 in the present sample was excellent (Cronbach's alpha $=.93$ ). Functional impairment was assessed using the Work and Social Adjustment Scale (WSAS), which rates impairment using a nine-point Likert scale (0-8) across five domains: work, home management, social life, private leisure activities, and family relationships (Mundt, Marks, Shear, \& Greist, 2002). The reliability of the WSAS in the present sample was excellent (Cronbach's alpha $=.92$ ).

Therapists assigned to the FIT group reviewed computerized feedback graphs for all of their patients, at the start of each therapy session, which classified their patients as "on track" or "not on track" on the PHQ-9 and GAD-7 measures. No feedback classifications were available for the WSAS, which was used as an independent control measure.

\subsection{Sample characteristics}

All patients who received at least two sessions of individual therapy with participating therapists during a one-year study period were included in the trial sample, including completers and drop outs. Patients attending group therapies were excluded; and those who only attended a single therapy session were excluded since the FIT technology only starts to provide feedback signals after session 1. Most participating patients were white British (89\%) females (66\%), with a mean age of 39.22 ( $S D=15.02$ ). Primary problems recorded in clinical records included affective disorders (35\%), generalized anxiety disorder (15\%), mixed anxiety and depressive disorder (14\%), and other common mental health problems. These diagnostic labels were established by psychological wellbeing practitioners using semi-structured assessment interviews at the time of initial referral to services, aided by a battery of validated screening measures for various common mental disorders (National Collaborating Centre for Mental Health, 2018). 


\subsection{Health economic analysis}

The cost-effectiveness analysis was based on health services costs and patient-reported health outcomes. Health services costs included the cost of training therapists $(k=39)$ who were randomly assigned to the FIT arm of the trial, and the overall cost of therapy sessions received by all patients in the trial. The cost of training therapists in the FIT group included the following. (A) Trainer cost: training was conducted for all participating sites by one senior psychotherapist who has expertise in using the FIT intervention (cost per hour based on NHS band 7 pay scale); this senior psychotherapist spent a total of $45.5 \mathrm{~h}$ across all sites ( $6.5 \mathrm{~h}$ per site times 7 sites, since 2 sites attended the same training day). (B) Trainee cost: this included the time spent by the 39 therapists who attended the FIT training sessions; each therapist attended one session, lasting $6.5 \mathrm{~h}$ (cost per hour based on NHS band 6 pay scale). (C) Preparation cost: the trainer spent $13 \mathrm{~h}$ altogether to prepare training materials, and also incurred expenses per training session (e.g., travel costs, printing training materials) of $£ 100$ per session. NHS pay scales were based on the Unit Costs of Health and Social Care (Curtis \& Burns, 2018). The total cost of training (i.e. the sum of items A-C above) was split and evenly added to the cost of treatment for all patients in the FIT arm $(n=$ 1176).

Next, the cost of therapy sessions received by each patient was estimated by multiplying each patient's total contact time with trial therapists by the hourly cost of therapists' time (Curtis \& Burns, 2018). No additional time was required to deliver the intervention because therapists were trained to incorporate FIT procedures within the allocated time for therapy sessions.

The primary outcome used in the cost-effectiveness analysis was reliable improvement in at least one of the two outcome measures (PHQ9 for depression and GAD-7 for anxiety), as long as the other measure did not show reliable deterioration. Reliable improvement was defined as an improvement of $\geq 6$ points on the PHQ- 9 and/or an improvement of $\geq 5$ points on the GAD-7 questionnaire, between baseline and posttreatment assessment scores.

Multilevel mixed-effects regression models were used to account for the nesting of patients within therapists. Each model had two levels, with patients at level 1 and therapists at level 2. The cost model was estimated with an identity link function, while the outcome model (reliable improvement) was estimated using a logistic link function. Therapists were specified as random effects in the model while covariates were modelled as fixed effects. The covariance matrix for random effects was specified as unstructured to avoid imposing any constraints. Baseline PHQ-9 and GAD-7 symptom severity measures and age were used as covariates to adjust for relevant clinical and demographic features. The models were estimated using 1000 bootstrap samples (Briggs, Wonderling, \& Mooney, 1997). The models were used to predict covariate-adjusted cost and probability of reliable improvement for the FIT and usual care arms. Next, an incremental cost-effectiveness ratio (ICER) was computed as a ratio of the difference in cost and the probability of reliable improvement. The ICER value represents the incremental cost of the FIT intervention (compared to usual care) per additional case of reliable improvement. Uncertainty in the ICER (based on the bootstrap samples) was plotted on a cost-effectiveness plane (Fig. 1). Finally, using the bootstrap samples, a cost-effectiveness acceptability curve (CEAC) was plotted, which shows the probability of FIT being cost-effective against a range of willingness-to-pay thresholds per additional case of reliable improvement (Fig. 2).

We also conducted a sensitivity analysis by re-estimating the models excluding outlier cases with unusually high costs of treatment (i.e., participants with cost of $>£ 1000$ were excluded, $n=16$ ). Finally, we reestimated the model by adding interaction terms for FIT and PHQ-9 score and FIT and GAD-7 score, to examine if the association between FIT and the outcome of interest (reliable improvement) was moderated by intake symptom severity.

Between-group comparisons regarding overall treatment duration

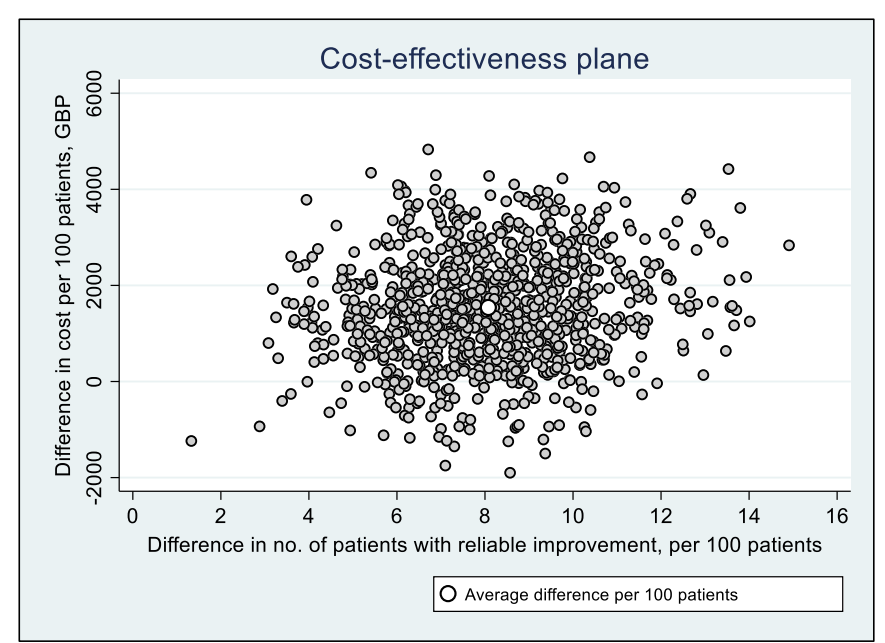

Fig. 1. Cost-effectiveness plane showing additional numbers of patients in the feedback-informed treatment group (out of 100) experiencing reliable improvement plotted against incremental cost, compared to usual care.

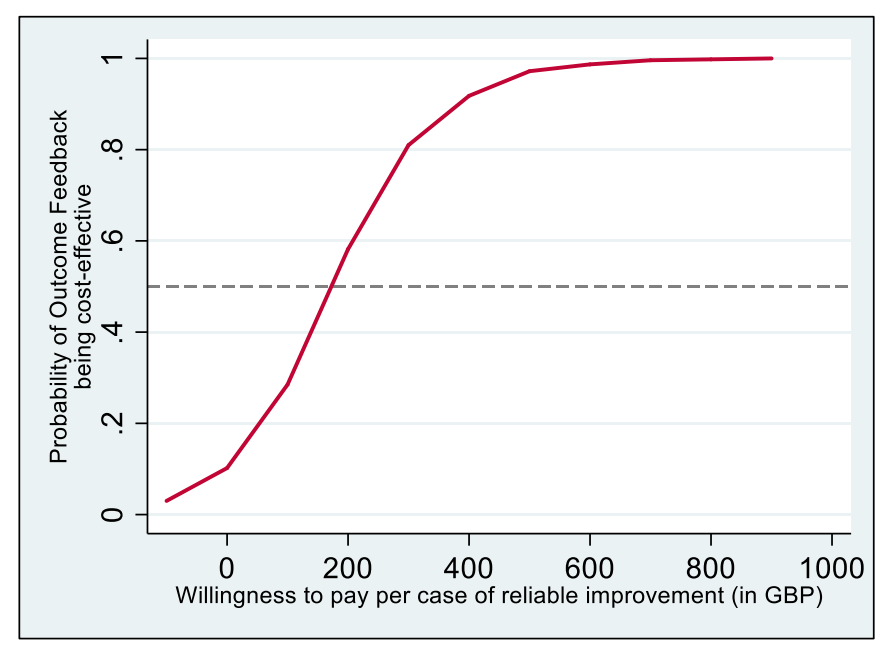

Fig. 2. Cost-effectiveness acceptability curve showing the probability of feedback-informed treatment being cost-effective for a range of willingness-topay values per case of reliable improvement.

and dropout rates have been presented in a prior publication (Delgadillo et al., 2018) and are therefore not repeated in the present study.

\section{Results}

Table 1 presents the results of mixed-effects regression models for treatment outcomes (reliable improvement) and costs. In the treatment outcome model, FIT was associated with an odds ratio of 1.23 relative to usual care $(95 \% \mathrm{CI}=0.976$ to $1.512, \mathrm{p}=0.067)$. This model was used to predict the covariate-adjusted probability of reliable improvement in the FIT and usual care arms. The difference in predicted probability (FIT minus usual care group) was 8.09 percentage points (95\% CI: $4.16 \%-$ $12.03 \%, \mathrm{p}<0.01$ ) and was statistically significant. The adjusted treatment cost for an average case (treatment episode for one patient) in the usual care group was $£ 273.50$. In the treatment cost regression model, the intervention group was associated with a marginally higher cost of $£ 15.17$ (95\% CI: $£ 6.95$ to $£ 37.29$ ) per case, after adjusting for baseline confounders, but the $95 \%$ confidence interval overlapped zero implying that the cost difference could range from $-£ 7.00$ to $£ 37.30$ (note that negative value here implies FIT may be cost-saving). In summary, patients in the FIT group had a statistically significant higher probability of 
Table 1

Results of mixed-effects regression models for reliable improvement and patient-level cost ${ }^{\mathrm{a}}$.

\begin{tabular}{|c|c|c|c|c|c|c|}
\hline Outcome: reliable improvement & Odds ratio & $\mathrm{SE}^{ \pm}$ & $\mathbf{Z}$ & $\mathbf{P}$ & $95 \% \mathrm{CI}$ & \\
\hline Feedback-informed treatment group & 1.222 & 0.136 & 1.83 & 0.067 & 0.976 & 1.512 \\
\hline Baseline PHQ-9 score & 1.012 & 0.011 & 0.94 & 0.348 & 0.991 & 1.033 \\
\hline Baseline GAD-7 score & 1.096 & 0.015 & 6.68 & $<0.01$ & 1.068 & 1.125 \\
\hline Age (years) & 1.009 & 0.003 & 2.39 & 0.017 & 1.002 & 1.016 \\
\hline Variance (therapist-level) & 0.374 & 0.099 & - & - & 0.222 & 0.623 \\
\hline Intra-cluster correlation & 0.102 & 0.024 & - & - & 0.063 & 0.160 \\
\hline Outcome: patient-level cost & Coefficient & $\mathrm{SE}^{ \pm}$ & $\mathrm{Z}$ & $\mathbf{P}$ & $95 \% \mathrm{CI}$ & \\
\hline Feedback-informed treatment group & 15.170 & 11.288 & 1.340 & 0.179 & -6.954 & 37.294 \\
\hline Baseline PHQ-9 score & -0.823 & 0.822 & -1.000 & 0.317 & -2.434 & 0.788 \\
\hline Baseline GAD-7 score & -0.038 & 1.011 & -0.040 & 0.970 & -2.019 & 1.944 \\
\hline Age (years) & 0.074 & 0.240 & 0.310 & 0.758 & -0.397 & 0.544 \\
\hline Variance (therapist-level) & 24189.3 & 4210.6 & - & - & 17197.1 & 34024.6 \\
\hline Variance (residual) & 29127.7 & 886.8 & - & - & 27440.4 & 30918.8 \\
\hline Intra-cluster correlation & 0.454 & 0.044 & - & - & 0.370 & 0.540 \\
\hline
\end{tabular}

${ }^{ \pm}$SE (standard error) and CI (confidence intervals) estimated using 1000 bootstrap samples.

${ }^{\text {a }}$ Multi-level structure was used, with patients nested within therapists.

reliable improvement compared to patients in the usual care group and may be associated with a small (but uncertain) incremental cost.

We also investigated interactions between group (FIT vs. usual care) and baseline severity in PHQ-9 and GAD-7 measures. However, these interaction terms were not statistically significant in the cost or treatment outcome models (see Appendix Table A1). Furthermore, we investigated the impact of removing extreme outliers (high-cost cases incurring $>£ 1000 ; n=16$ ). The results were robust even after excluding outliers, as the odds ratio for reliable improvement and the incremental cost of FIT remained stable (albeit the magnitude of coefficients were marginally more favourable to FIT) (Appendix Table A2).

For health services decision-making, it is important to evaluate the joint distribution of incremental costs and incremental probability of a relevant clinical outcome (e.g., reliable improvement). Fig. 1 presents a cost-effectiveness plane which models outcomes for 100 patients, showing the difference between the FIT and usual care groups in the number of patients with reliable improvement (x-axis) and the difference in treatment costs (y-axis). The white dot in the middle of the figure represents the average difference per 100 patients. For 100 patients treated with FIT, the intervention would produce 8.1 additional patients (95\% CI: 4.2 to 12.0 ) with reliable improvement at the cost of $£ 1517$ (95\% CI: $£ 695$ to $£ 3729$ ), compared to usual care. The incremental costeffectiveness ratio (ICER) per additional case of reliable improvement, calculated as the ratio of difference in cost and difference in cases with reliable improvement, was $£ 187.40$ (95\% CI: $£ 126.70$ to $£ 501.50$ ). This confidence interval around the ICER shows that the economic value of the FIT intervention may range from being a dominant strategy (i.e., one that improves health outcomes and saves cost) to a strategy that improves outcomes for a modest increase in cost. Finally, the decision uncertainty is presented probabilistically in the form of a CEAC (Fig. 2) which assesses the joint distribution of incremental costs and reliable improvement. The CEAC shows the probability (y-axis) of FIT being cost-effective for a range of willingness-to-pay (WTP, x-axis) thresholds. The probability of FIT being cost-effective is $50 \%$ at the mean value of ICER - this probability increases to $90 \%$ if the health system is willing to pay an additional cost of $£ 370$ per case of reliable improvement.

\section{Discussion}

This study presents the first health economic evaluation of FIT to date, using data from the largest multi-service clinical trial of feedback technology conducted in mainstream, outpatient psychological therapy services. Our findings indicate that FIT is likely to be associated with a small (and uncertain) incremental cost per treatment episode, which increased the probability of reliable symptomatic improvement by approximately $8 \%$ (95\% CI: 4\%-12\%). For every 100 patients accessing psychological care, FIT would produce one additional case of reliable improvement at an incremental cost of $£ 187.4$ (95\% CI: $£ 126.7$ to $£ 501.5$ ), representing a modest expense to the health service, although the uncertainty interval shows that FIT may in fact be a cost-saving strategy. The potential additional cost takes into consideration the training costs incurred to support the competent use of the FIT technology by therapists assigned to the experimental group. As this cost is incurred only once, it is considerably offset by its clinical benefits as trained therapists treat additional patients using the FIT approach over a longer time horizon. Furthermore, this training cost could be reduced in the future by supporting the training of future FIT technology-users through an online e-learning module, instead of incurring the costs of expert trainers. Overall, our probabilistic analysis (based on the CEAC) indicates that integrating FIT technology in routine psychological care would improve the overall cost-effectiveness of treatment if health services were willing to pay a modest incremental cost per treatment episode, and this cost is offset by treatment gains over time. Furthermore, these outcomes are not confounded by treatment utilisation indices, since the mean number of treatment sessions and dropout rates were not significantly different between groups (Delgadillo et al., 2018).

This study has several strengths, including the rigorous comparison of FIT vs. usual care using a cluster randomised controlled trial design, the large sample size, the broad inclusion/exclusion criteria and multisite design to maximise generalisability to mainstream psychological services in England. Nevertheless, it is important to interpret these findings in light of some limitations. Firstly, data on utility-based instruments were not available to allow estimation of quality-adjusted life years (QALYs) for a cost-utility analysis. Similarly, we had no data on other relevant health economic indices such as additional healthcare utilisation, or work participation. However, for a decision-maker in the mental health sector, reliable improvement is a clinically relevant outcome for resource allocation decisions, since it recognises that not all patients attain full remission of symptoms but nevertheless benefit from symptom reductions attributable to treatment. Secondly, the primary outcomes were assessed at the end of the acute-phase of treatment, and no follow-up assessments were conducted, so the long-term sustainability of the observed outcomes is unknown. Thirdly, the cost data did not include other primary and secondary care resource use; however, this is a conservative approach implying that the incremental cost of the intervention may be overestimated. Furthermore, as in most progress feedback studies, only patient-reported measures were used to provide feedback and to determine clinical outcomes, and there was an absence 
of clinician-rated outcomes or structured diagnostic interviews. Independent diagnostic interviews would be an ideal feature of feedback trials, but the cost of conducting such interviews with thousands of trial participants would be prohibitively steep. In this regard, the use of selfreported psychometric measures is a pragmatic and valid approach. One way to enhance the rigour of pragmatic trials that rely on self-reported questionnaires is to introduce an independent control measure that is not used to provide feedback, but simply to measure an associated outcome. Although this trial was limited by a lack of independent diagnostic interviews, the beneficial effects of feedback were consistent across a range of relevant outcome domains (depression, anxiety), including functional impairment (WSAS) which was introduced as an independent control measure (Delgadillo et al., 2018).

To date, there is convincing evidence from several randomised controlled trials of FIT systems implemented around the world, indicating that using routine outcome monitoring and feedback technology improves the effectiveness of treatment, resulting in a small incremental effect size over-and-above usual psychological treatment (e.g., De Jong et al., 2021; Kendrick et al., 2016; Knaup et al., 2009; Lambert et al., 2018; Shimokawa et al., 2010). This study adds to this literature by demonstrating that FIT also improves the cost-effectiveness of psychological treatment for common mental disorders, at a modest incremental expense to healthcare purchasers.

\section{Funding}

This article describes work conducted under Grow MedTech's Proof of Concept programme, supported by UKRI Research England's Connecting Capability Fund [project code: CCF11-7795].

\section{Data sharing policy}

In line with the requirements of the ethics review board for this study, requests for access to data are to be made in writing to the corresponding author.

\section{CRediT authorship contribution statement}

Jaime Delgadillo: Conceptualization, Data curation, Funding acquisition, Methodology, Writing - original draft, Writing - review \& editing. Dean McMillan: Conceptualization, Funding acquisition, Supervision, Writing - review \& editing. Simon Gilbody: Conceptualization, Funding acquisition, Supervision, Writing - review \& editing. Kim de Jong: Conceptualization, Methodology, Writing - review \& editing. Mike Lucock: Conceptualization, Methodology, Writing - review \& editing. Wolfgang Lutz: Conceptualization, Methodology, Writing review \& editing. Julian Rubel: Conceptualization, Methodology, Writing - review \& editing. Elisa Aguirre: Conceptualization, Methodology, Writing - review \& editing. Shehzad Ali: Conceptualization, Data curation, Formal analysis, Methodology, Writing - original draft, Writing - review \& editing.

\section{Declaration of competing interest}

None.

\section{Appendix A. Supplementary data}

Supplementary data to this article can be found online at https://doi. org/10.1016/j.brat.2021.103873.

\section{References}

Briggs, A. H., Wonderling, D. E., \& Mooney, C. Z. (1997). Pulling cost-effectiveness analysis up by its bootstraps: A non-parametric approach to confidence interval estimation. Health Economics, 6(4), 327-340. https://doi.org/10.1002/(SICI)10991050(199707)6:4\%3C327::AID-HEC282\%3E3.0.CO;2-W.

Curtis, L., \& Burns, A. (2018). Unit costs of health and social care 2019. Canterbury: Personal Social Services Research Unit, University of Kent. https://doi.org/ 10.22024/UniKent/01.02.79286.

De Jong, K., Conijn, J. M., Gallagher, R. A. V., Reshetnikova, A. S., Heij, M., \& Lutz, M. C. (2021). Using progress feedback to improve outcomes and reduce drop-out, treatment duration, and deterioration: A multilevel meta-analysis. Clinical Psychology Review, 85, 102002. https://doi.org/10.1016/j.cpr.2021.102002.

Delgadillo, J., de Jong, K., Lucock, M., Lutz, W., Rubel, J., Gilbody, S., et al. (2018). Feedback-informed treatment versus usual psychological treatment for depression and anxiety: A multisite, open-label, cluster randomised controlled trial. The Lancet Psychiatry, 5(7), 564-572. https://doi.org/10.1016/S2215-0366(18)30162-7.

Delgadillo, J., Overend, K., Lucock, M., Groom, M., Kirby, N., McMillan, D., et al. (2017). Improving the efficiency of psychological treatment using outcome feedback technology. Behaviour Research and Therapy, 99, 89-97. https://doi.org/10.1016/j. brat.2017.09.011.

Janse, P. D., De Jong, K., Van Dijk, M. K., Hutschemaekers, G. J., \& Verbraak, M. J. (2017). Improving the efficiency of cognitive-behavioural therapy by using formal client feedback. Psychotherapy Research, 27(5), 525-538. https://doi.org/10.1080/ 10503307.2016.1152408.

Kendrick, T., El-Gohary, M., Stuart, B., Gilbody, S., Churchill, R., Aiken, L., et al. (2016). Routine use of patient reported outcome measures (PROMs) for improving treatment of common mental health disorders in adults. Cochrane Database of Systematic Reviews, 7, Article CD011119. https://doi.org/10.1002/14651858.CD011119.pub2.

Knaup, C., Koesters, M., Schoefer, D., Becker, T., \& Puschner, B. (2009). Effect of feedback of treatment outcome in specialist mental healthcare: meta-analysis. The British Journal of Psychiatry, 195(1), 15-22. https://doi.org/10.1192/bjp. bp.108.053967.

Kroenke, K., Spitzer, R. L., \& Williams, J. B. W. (2001). The PHQ-9: Validity of a brief depression severity measure. Journal of General Internal Medicine, 16(9), 606-613. https://doi.org/10.1046/j.1525-1497.2001.016009606.x.

Kroenke, K., Spitzer, R. L., Williams, J. B. W., Monahan, P. O., \& Löwe, B. (2007). Anxiety disorders in primary care: Prevalence, impairment, comorbidity, and detection. Annals of Internal Medicine, 146(5), 317-325. https://doi.org/10.7326/0003-4819146-5-200703060-00004.

Lambert, M. J., Hansen, N. B., \& Finch, A. E. (2001). Patient-focused research: Using patient outcome data to enhance treatment effects. Journal of Consulting and Clinical Psychology, 69(2), 159-172. https://psycnet.apa.org/doi/10.1037/0022-006X .69 .2 .159 .

Lambert, M. J., Whipple, J. L., \& Kleinstäuber, M. (2018). Collecting and delivering progress feedback: A meta-analysis of routine outcome monitoring. Psychotherapy, 55(4), 520-537. https://doi.org/10.1037/pst0000167.

Mundt, J. C., Marks, I. M., Shear, M. K., \& Greist, J. M. (2002). The work and social adjustment scale: A simple measure of impairment in functioning. British Journal of Psychiatry, 180(5), 461-464. https://doi.org/10.1192/bjp.180.5.461.

National Collaborating Centre for Mental Health. (2018). The Improving Access to Psychological Therapies Manual. Retrieved from https://www.england.nhs.uk/wp -content/uploads/2018/06/the-iapt-manual.pdf.

National IAPT Team. (2015). National curriculum for the education of psychological wellbeing practitioners (3rd ed.). London: NHS England/Department of Health.

National Institute for Health and Care Excellence. (2011). Common mental health problems: Identification and pathways to care. London: National Collaborating Centre for Mental Health.

Richards, D. A., \& Borglin, G. (2011). Implementation of psychological therapies for anxiety and depression in routine practice: Two year prospective cohort study. Journal of Affective Disorders, 133(1-2), 51-60. https://doi.org/10.1016/J. JAD. 2011.03.024.

Roth, A. D., \& Pilling, S. (2008). Using an evidence-based methodology to identify the competences required to deliver effective cognitive and behavioural therapy for depression and anxiety disorders. Behavioural and Cognitive Psychotherapy, 36(2), 129-147. https://doi.org/10.1017/S1352465808004141.

Shimokawa, K., Lambert, M. J., \& Smart, D. W. (2010). Enhancing treatment outcome of patients at risk of treatment failure: meta-analytic and mega-analytic review of a psychotherapy quality assurance system. Journal of Consulting and Clinical Psychology, 78(3), 298-311. https://psycnet.apa.org/doi/10.1037/a0019247. 